\title{
Efecto de una dieta sin antibióticos, coccidiostatos y aminoácidos sintéticos en pollos sexados Cobb 500
}

\author{
Effect of a diet free of antibiotics, coccidiostats and synthetic \\ amino acids in Cobb 500 sexed chickens.
}

\author{
Angel Roberto Sánchez Quinche ${ }^{1}$ \\ Carla Dennisse Muñoz Izquierdo ${ }^{2}$ \\ Juan Marcelo Jurado Correa ${ }^{3}$ \\ Erika María León Armijos ${ }^{4}$ \\ Dioselina Esmeralda Pimbosa Ortiz
}

DOI: https://doi.org/10.19053/01228420.v18.n3.2021.12744

RESUMEN: La presente investigación se desarrolló en el área experimental de la Granja Santa Inés perteneciente a la Facultad de Ciencias Agropecuarias de la Universidad Técnica de Machala-Ecuador, con la finalidad de evaluar el efecto de una dieta sin antibióticos, coccidiostatos y aminoácidos sintéticos, administrada a pollos Cobb 500 sexados sobre los parámetros productivos: Peso vivo, consumo de agua y alimento acumulado, conversión alimenticia, mortalidad, peso ante mortem, peso de la canal y porcentaje de rendimiento de la canal. Se empleó un DCA, en el cual se utilizó un único factor a manipular, el balanceado, separando los machos de las hembras, de una muestra de 100 aves, aplicando una réplica por sexo, dando como resultado 4 unidades experimentales (jaulas), con 25 unidades muestrales (pollos) por replica, las jaulas fueron distribuidas con un número 1 y 4 (hembras), 2 y 3 (machos datos se procesaron semanalmente. Para el análisis estadístico, se empleó el programa Statgraphics Centurión XV.I. ${ }^{\oplus}$, aplicando un ANOVA y para establecer las diferencias entre las medias se usó el procedimiento de comparación múltiple de Bonferroni con un nivel del $95,0 \%$ de confianza. Los resultados muestran que los machos a partir de la 2da semana presentan los mejores pesos, así como el mejor peso de la canal, mientras que en el porcentaje de rendimiento de la canal y consumo de agua de bebida destacan las hembras sobre todo en los últimos días del experimento. Demostrando un efecto marcado en algunas variables, al someter a los sexos a una dieta especial.

PALABRAS CLAVE: Balanceado libre de APC; peso vivo; consumo de alimento; consumo de agua; índice de conversión.
ABSTRACT: This research was carried out in the experimental area of the Santa Inés Farm belonging to the Faculty of Agricultural Sciences of the Technical University of Machala-Ecuador, with the purpose of evaluating the effect of a diet without antibiotics, coccidiostats and synthetic amino acids, administered to sexed Cobb 500 chickens on the productive parameters: live weight, water and accumulated feed consumption, feed conversion, mortality, ante-mortem weight, carcass weight andcarcass yield percentage. A DCA was used, in which only one factor to manipulate was used, the balance, separating males from females, from a sample of 100 birds, applying one replicate per sex, resulting in 4 experimental units (cages), with 25 sample units (chickens) per replicate, the cages were distributed with a number 1 and 4 (females), 2 and 3 (males), whose data were processed weekly. For the statistical analysis, the Statgraphics Centurión XV.I. ${ }^{\oplus}$ program was used, applying an ANOVA and to establish the differences between means, the Bonferroni multiple comparison procedure was used with a $95.0 \%$ confidence level. The results show that the males from the 2 nd week onwards present the best weights, as well as the best carcass weight, while in the percentage of carcass yield and drinking water consumption, the females stand out, especially in the last days of the experiment. Showing a marked effect in some variables, when the sexes were subjected to a special diet.

KEYWORD: GPA-free feed; live weight; feed consumption; water consumption; feed conversion index.

CITAR COMO: Sánchez Quinche, A. R., Muñoz Izquierdo , C. D., Jurado Correa , J. M., León Armijos , E. M., \& Pimbosa Ortiz, D. E. Efecto de una dieta sin antibióticos, coccidiostatos y aminoácidos sintéticos en pollos sexados Cobb 500. Cien. Agri., 18(3): 63-77. https://doi.org/10.19053/01228420.v18. n3.2021.12744

1 Universidad Técnica de Machala - Grupo de Investigación en Producción de Alimentos y Sanidad Animal (GIPASA). Semillero de Investigación en Producción Animal (SIPA). (Machala -Ecuador), arsanchez@utmachala.edu.ec (D) ORCID: 0000-0002-3582-1656

2 Universidad Técnica de Machala - SIPA(Machala-Ecuador), cmunoz3@utmachala.edu.ec (D) ORCID: 0000-0002-7073-9762

3 Universidad Técnica de Machala - SIPA(Machala-Ecuador),jmjurado_est@utmachala.edu.ec (D) ORCID: 0000-0003-4837-5736

4 Universidad Técnica de Machala - SIPA(Machala-Ecuador), eleon2@utmachala.edu.ec (D) ORCID: 0000-0001-9508-5817

5 Universidad Técnica de Machala - GIPASA - SIPA (Machala-Ecuador),dpimbosa@utmachala.edu.ec (D) ORCID: 0000-0001-6146-1845 


\section{INTRODUCCIÓN}

En la industria avícola, el balanceado es de vital importancia para conseguir el crecimiento y desarrollo deseado de las aves, donde resalta la calidad de las materias primas para alcanzar el objetivo productivo, ya que el tipo de alimentación que recibe el animal, tendrá un efecto en la integridad intestinal, es decir, permitiendo una mayor o menor absorción de nutrientes, Osorio et al. (2010).

Hegde et al. (2016) manifiestan que los antibióticos utilizados normalmente en las dietas de las aves influyen en la microbiota intestinal al causar problemas en los rendimientos productivos por la disminución de las poblaciones bacterianas benéficas, disminuyendo la asimilación de nutrientes, además de la posible presencia de residuos en la carne, perjudicando la salud del consumidor por la posible aparición de bacterias resistentes a los mismos.

Kumar et al. (2018) manifiestan la gran problemática en la producción animal "el uso indiscriminado de antibióticos", utilizados como subterapias para mejorar la asimilación de nutrientes, incrementar el crecimiento y desarrollo de las aves; además, en pollos broilers, utilizados para controlar y prevenir la enteritis necrótica, sin embargo, estos presentan grandes desventajas, siendo la más preocupante, la resistencia bacteriana.

Applegate (2017), menciona que la eliminación de antibióticos subterapéuticos de las dietas de aves de corral en Europa y en otras partes del mundo ha despertado interés corporativo y académico en mejorar la salud intestinal, la utilización de nutrientes y reducir sus pérdidas debido a respuestas inmunes innatas, por lo que, los probióticos, prebióticos, ácidos orgánicos, ácidos grasos de cadena corta / media y aditivos para piensos de extractos de plantas están ganando presencia en el mercado, siendo prometedores para actuar sobre varios factores estresantes del ave, influyendo en el rendimiento y la uniformidad, por lo que se hace imperativo comprender y aplicar estas estrategias en beneficio del mantenimiento y la eficiencia alimenticia.

Las dietas que no llevan antibióticos, coccidiostatos y aminoácidos sintéticos tienden a ser menos eficaces que aquellas en las cuales, se utilizan todos los aditivos que contiene un alimento comercial, sin embargo, la demanda del mercado actual exige la producción de alimentos inocuos, por lo tanto, la normativa de cada país, en este caso, órgano del gobierno del Ecuador (Agrocalidad, 2017) en la Norma 7,5 artículo 30, describe textualmente que: "en el caso de que se añadan antibióticos para tratamiento a las fórmulas balanceadas, esta acción deberá estar sujeta a la prescripción y supervisión del médico veterinario responsable y registrar su uso, en este caso se debe tomar en consideración los tiempos de retiro del uso de antibióticos antes del faenamiento de las aves", sin embargo, en numerosas ocasiones esta norma no es respetada, debido a que los productores desconocen de los tiempos de retiro o el manejo del fármaco utilizado para el tratamiento antibacteriano 
o anticoccidiosis, provocando problemas en la salud pública. Debido a esto, se requiere del mejoramiento de las dietas alternativas que eviten el uso de estos productos, como ejemplo tenemos los anticoccidiales químicossintéticos que al ser mal manejados podrían terminar siendo perjudiciales para el consumidor y a su vez brinden un producto cárnico de calidad, al ser obtenido con una mezcla alimenticia eficiente e inocua, permitiendo reducir los costos de producción (Gutiérrez et al., 2013; Gerardo et al., 2020).

Además, según Shang et al. (2018), se debe contemplar la influencia de las materias primas y aditivos sobre la microbiota intestinal, por ejemplo, el maíz, produce un bajo porcentaje de enterococos, clostridios y lactobacilos C y G, el trigo influye en mayores porcentajes de bífidobacterias $\mathrm{C}$ y $\mathrm{G}$, por lo que su utilización en la formulación de raciones ecológicas es de gran beneficio para incrementar los rendimientos productivos. Sin embargo, Valenzuela et al. (2015), manifiesta que, los alimentos concentrados proteicos utilizados en dietas de pollos de engorde, son considerados de alto costo, siendo las fuentes proteicas más usadas los subproductos de oleaginosas como harina y afrecho de soya, debido a su alto contenido en proteína digestible y equilibrado perfil de aminoácidos.

Según Pindo \& Sánchez (2016), un balanceado ecológico cubrirá los requerimientos proteicos con la inclusión de harina de soya y pescado, la energía a través de fuentes de almidones como maíz, y fuentes de ácidos grasos como el aceite de soya en inicio y aceite de palma en etapa de crecimiento y engorde y la zeolita como atrapante de toxinas. Pérez et al. (2019) menciona que como ingreso de minerales se utilizan carbonato de calcio y fósforo; mientras que como fuente de coccidiostatosy control bacteriano se emplean plantas medicinales como Lippia alba (mastrante), Ocimum basilicum (albahaca), Cymbopogon citratus (hierba luisa) y Plectranthus amboinicus (oréganon), (Chiriboga et al. 2015; Caivinagua, 2016; Sánchez et al. 2016; Rueda, 2017).

El objetivo de esta investigación fue evaluar el efecto de una dieta sin antibióticos, coccidiostatos y aminoácidos sintéticos administrada a pollos Cobb 500 sexados sobre los parámetros productivos: peso vivo, consumo de agua y alimento acumulado, conversión alimenticia, mortalidad, peso ante mortem, peso de la canal y porcentaje de rendimiento de la canal.

\section{Materiales y Métodos}

\section{A. Localización de la investigación}

El experimento fue realizado en la granja Santa Inés de la Universidad Técnica de Machala, localizada en el kilómetro 5 1 12 vía Machala - Pasaje, longitud: 79 54' 05", latitud: 3¹7'16", altitud: 5 msnm, a una temperatura aproximada de 22 a $35^{\circ} \mathrm{C}$. En las figuras 1 y 2 se muestran los comportamientos de temperatura y de humedad relativa durante el tiempo de análisis. 


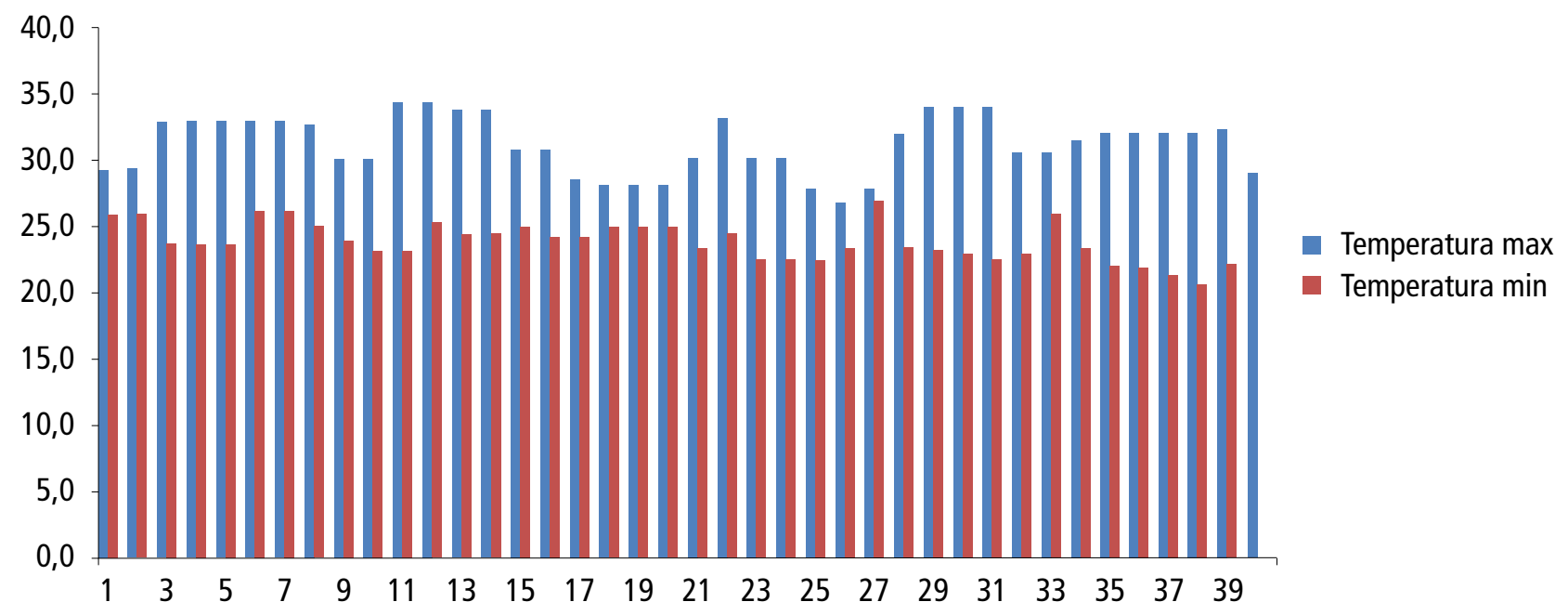

FIGURA 1. Registro de la temperatura en $\mathrm{C}^{\circ}$ tomado en la mañana (7:00 a.m.) durante el desarrollo del experimento, obtenida con un termohigrómetro digital marca: LWH modelo: HTC-2. Se puede apreciar los picos de temperatura máximos (azul) y mínimos (rojos) obtenidos durante toda la investigación.

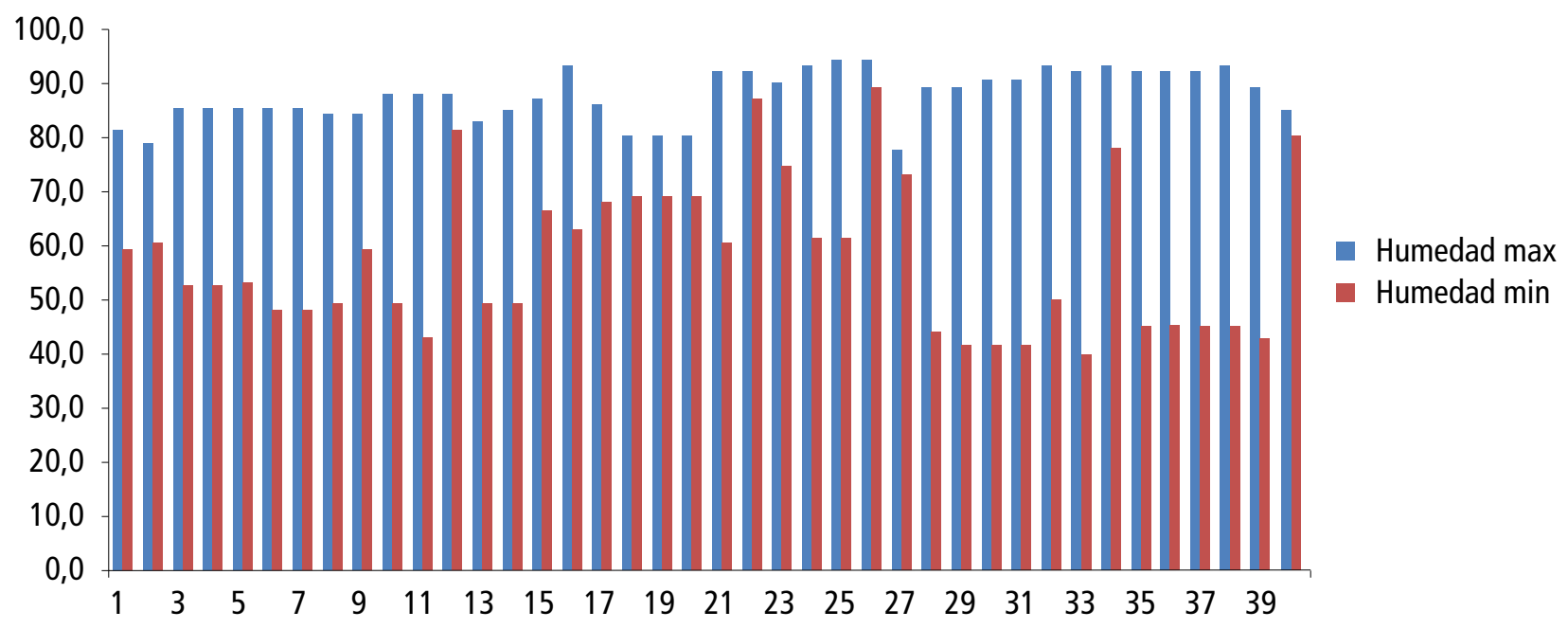

FIGURA 2. Registro de la humedad relativa de la nave expresada en porcentaje, mínimas y máximas de cada día por la mañana (7:00 a.m.) durante el desarrollo del experimento obtenida con un termohigrómetro digital marca: LWH modelo: HTC-2. Se puede apreciar los picos de humedad máximos (azul) y mínimos (rojos) obtenidos durante toda la investigación.

\section{B. Características de la nave}

Para el desarrollo de la investigación, se utilizó una nave, basado en las normas que contemplan Agrocalidad (2017) adaptado para 100 pollos broilers sexados de la línea Cobb 500; con una altura de techo de 2,5 m, con $10 \mathrm{~m}$ de largo y $5 \mathrm{~m}$ de ancho. Se instalaron cortinas plásticas de crianza y laterales para evitar las corrientes de aire y el excesivo enfriamiento, sobre todo en las primeras semanas de vida de las aves. Se realizó una adecuada limpieza y desinfección de las instalaciones, equipos y demás materiales, así como también el tratamiento de la yacija que se colocó sobre el piso de concreto, la cual era de viruta de madera gruesa. Para bajar la carga de patógenos, se utilizó formol-formaldehído en una dosis de $400 \mathrm{ml}$ de producto en 20 litros 
de agua para así evitar contaminantes microbiólogos que puedan existir y afectar negativamente al experimento. Llevando a cabo estos procedimientos antes de la llegada del lote de pollos y como fuente de calor se usaron focos amarillos de 100 watts en cada unidad experimental y una calentadora a gas para proporcionar la temperatura de confort.

\section{Preparación del Balanceado y Manejo del pollo}

Para la formulación de la mezcla, se utilizó la herramienta Solver de Excel siguiendo los valores tabulados de las materias primas publicados en las tablas FEDNA 2019 y De Blass et al. (2019), y como base para las fórmulas, se tomó los requerimientos nutricionales publicados en la tabla de Santoma \& Mateos (2018), asumiendo para el experimento tres fases: dieta inicial desde el día 0 al 21(dato de referencia, iniciación de 0 a 14 días de vida del pollo de engorde), cuyas materias primas fueron harina de pescado (59\%), harina de soja (44\%), maíz nacional, aceite de soja, rovabio (xilanasa, B-glucanasas, celulasas, pectinasas, proteasas, B-manosidasas), premezcla vitamínica-mineral (CUSTOM PACK BROILER), sal, carbonato de calcio, fosfato bicalcico Anh, Plectranthus amboinicus y zeolita. La dieta crecimiento a partir del día 22 al 35 (dato de referencia: Crecimiento de 15 -23 días), con la diferencia de que el aceite de palma sustituye al de soya y la planta medicinal es reemplazada por Lippia alba. Y la dieta de finalización a partir del día 35 en adelante (dato de referencia, ceba de 24 - 36 días), cuya característica es similar a la dieta de crecimiento, con la excepción de la eliminación de la harina de pescado. El procedimiento de la mezcla consistió en adicionar las materias primas de mayor proporción (macro mezcla), luego las de menor porcentaje de inclusión (micromezcla) y está añadida a la primera, y finalmente la adición de la zeolita para una homogeneización total.

TABLA 1. Valores nutricionales de las fases de alimento obtenidas con la herramienta Solver de Excel y usadas en el experimento.

\begin{tabular}{|l|c|c|c|}
\hline Valores nutricionales & $\begin{array}{c}\text { Balanceado } \\
\text { iniciación }\end{array}$ & $\begin{array}{c}\text { Balanceado } \\
\text { crecimiento }\end{array}$ & $\begin{array}{c}\text { Balanceado } \\
\text { Finalización }\end{array}$ \\
\hline Proteína Bruta (g/Kg) & 214,04 & 198,81 & 182,70 \\
\hline Fibra Bruta (g/Kg) & 33,08 & 31,82 & 30,93 \\
\hline Calcio $(\mathrm{g} / \mathrm{Kg})$ & 9,80 & 9,00 & 7,50 \\
\hline Fosforo $(\mathrm{g} / \mathrm{Kg})$ & 6,60 & 5,80 & 5,60 \\
\hline Sodio $(\mathrm{g} / \mathrm{Kg})$ & 1,90 & 1,70 & 1,60 \\
\hline Cloro $(\mathrm{g} / \mathrm{Kg})$ & 2,41 & 2,08 & 1,90 \\
\hline EMA $(\mathrm{Kcal} / \mathrm{Kg})$ & 2875,00 & 3015,53 & 3037,82 \\
\hline Lisina $(\mathrm{g} / \mathrm{Kg})$ & 12,04 & 10,95 & 9,84 \\
\hline Metionina + Cisteína $(\mathrm{g} / \mathrm{Kg})$ & 6,62 & 6,26 & 5,78 \\
\hline Treonina $(\mathrm{g} / \mathrm{Kg})$ & 8,26 & 7,65 & 7,00 \\
\hline
\end{tabular}


Cuatro horas antes al ingreso de los pollos a las jaulas se encendió la fuente de calor, se colocó sobre la cama papel periódico, de tal manera que quedó cubierta durante 3 días, así mismo durante ese tiempo se adiciono vitaminas más electrolitos en una dosis $1 \mathrm{~g}$ por litro de agua de bebida y el alimento de acuerdo al estandarizado de los objetivos de desempeño "como al nacimiento" de los pollos Cobb 500 (Cobb-Vantress, 2018). El manejo de la temperatura se realizó conforme al manual Cobb 500, retirándose la criadora a gas al día 10, y los focos amarillos cambiamos a blancos al día 21. El manejo de las cortinas se dio de tal manera que a partir del día 8 se dejó un espacio de $20 \mathrm{~cm}$ que iban aumentando en la misma proporción conforme pasaban los días, hasta que al día 15 se procedió a retirar la cortina interna (de crianza) de la misma manera, la cortina externa fue retirada en su totalidad al día 21. El programa de ciclo luminoso en las primeras semanas se mantuvo 24 horas y a partir del día 8 se fue retirando una hora por día, de tal manera que los animales llegaron a recibir, solo 6 horas de luz artificial en la madrugada. Se empleó un plan básico vacunal que se basó en inmunizar aplicando Gumboro D78 a los 5 días y su revacunación al día 15 y con Newcastle "La Sota" en el día 8 y en el día 23; las revacunaciones se realizaron en el agua de bebida.

Según Arcila-Lozano et al. (2004), las diversas variedades de orégano poseen diferentes actividades microbianas ya que presentan actividad en contra de las bacterias gram negativas y gram positivas además capacidad antifúngica. Para mantener la salud de los pollos y mejorar el sabor de la carne se adicionó $5 \mathrm{ml}$ de infusión de oreganón al 50\% por litro de agua de bebida desde el día 3 al 5 en la primera semana de vida, luego se administró durante 5 días consecutivos en la tercera y sexta semana de vida. Para contrarrestar la alcalosis producida en el animal por la alta temperatura de la zona, se usó 0,5 $\mathrm{ml}$ de vinagre/litro de agua de bebida a partir de la 3 semana en adelante.

Al día 23 de análisis, se administró $0,5 \mathrm{ml}$ de yodo (Tintura de Yodo Weir) por litro de agua de bebida como medida preventiva a la presencia de lesiones de la mucosa serosa de la molleja.

\section{Diseño experimental}

La investigación fue de tipo experimental, se basó en el argumento vigente de la Agencia de Regulación y Control Fito y Zoosanitario de la República del Ecuador, Almeida (2021), empleando un Diseño Completamente al Azar (DCA), en el cual se utilizó como tratamiento el balanceado carente de APC, coccidiostatos y aminoácidos sintéticos, como único factor a manipular, se procedió a separar los machos de las hembras de una muestra de 100 aves, aplicando una réplica por sexo, dando como resultado 4 unidades experimentales (jaulas), con 25 unidades muestrales (pollos) por replica. Las jaulas fueron distribuidas con un número 1 y 4 (hembras), 2 y 3 (machos). 
No se empleó un grupo control debido a que solo se consideró el efecto de alimentación en el sexo de los animales.

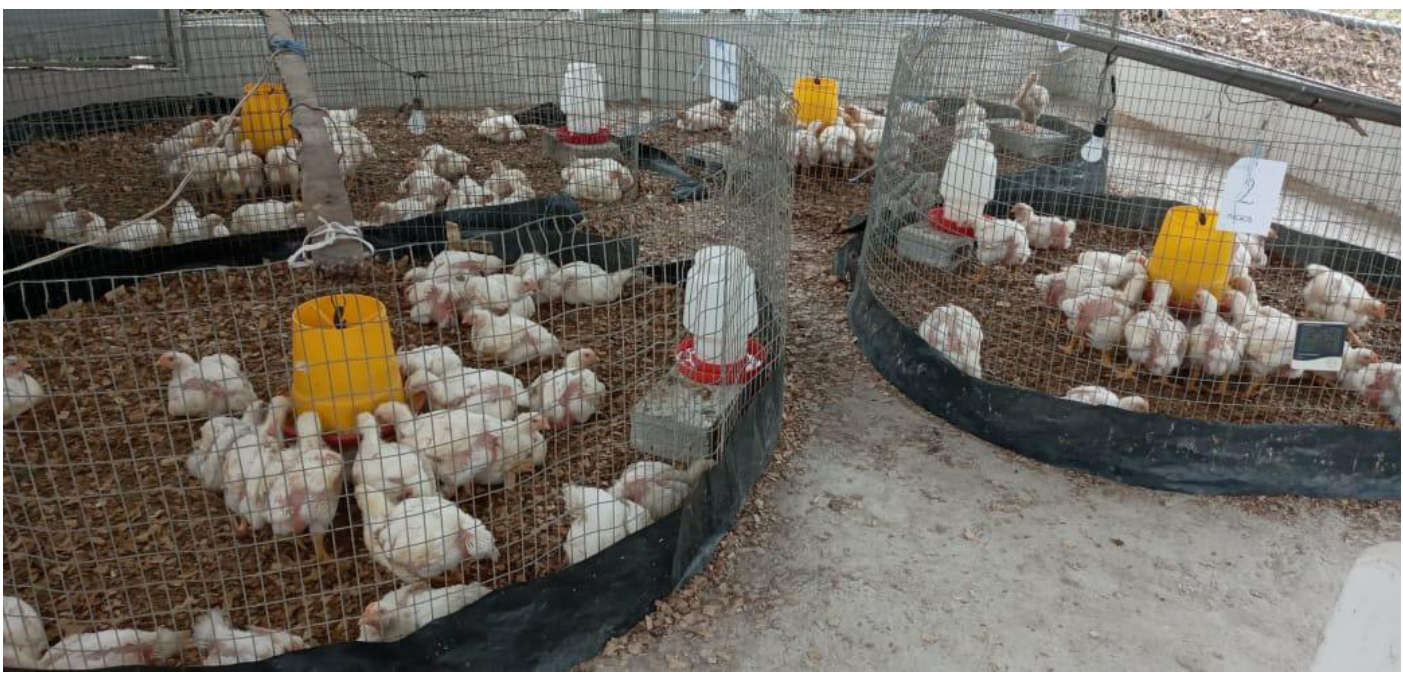

FIGURA 3. Distribución de las réplicas de los pollos Cobb 500 sexados.

\section{E. Variables evaluadas}

Peso vivo (g): Los pesos fueron tomados desde el día 0 de la recepción de los pollos con la ayuda de una balanza electrónica (CAMRY modelo EK9332-F302), con un margen de error de $\pm 1 \mathrm{~g}$. La toma se realizó semanalmente hasta el día 40 que se sacrificaron los animales, obteniéndose 600 datos (2 Sexos $x$ 2 Replicas x 25 Pollos x 6 Tiempos) sin descontar mortalidad presentada en la investigación.

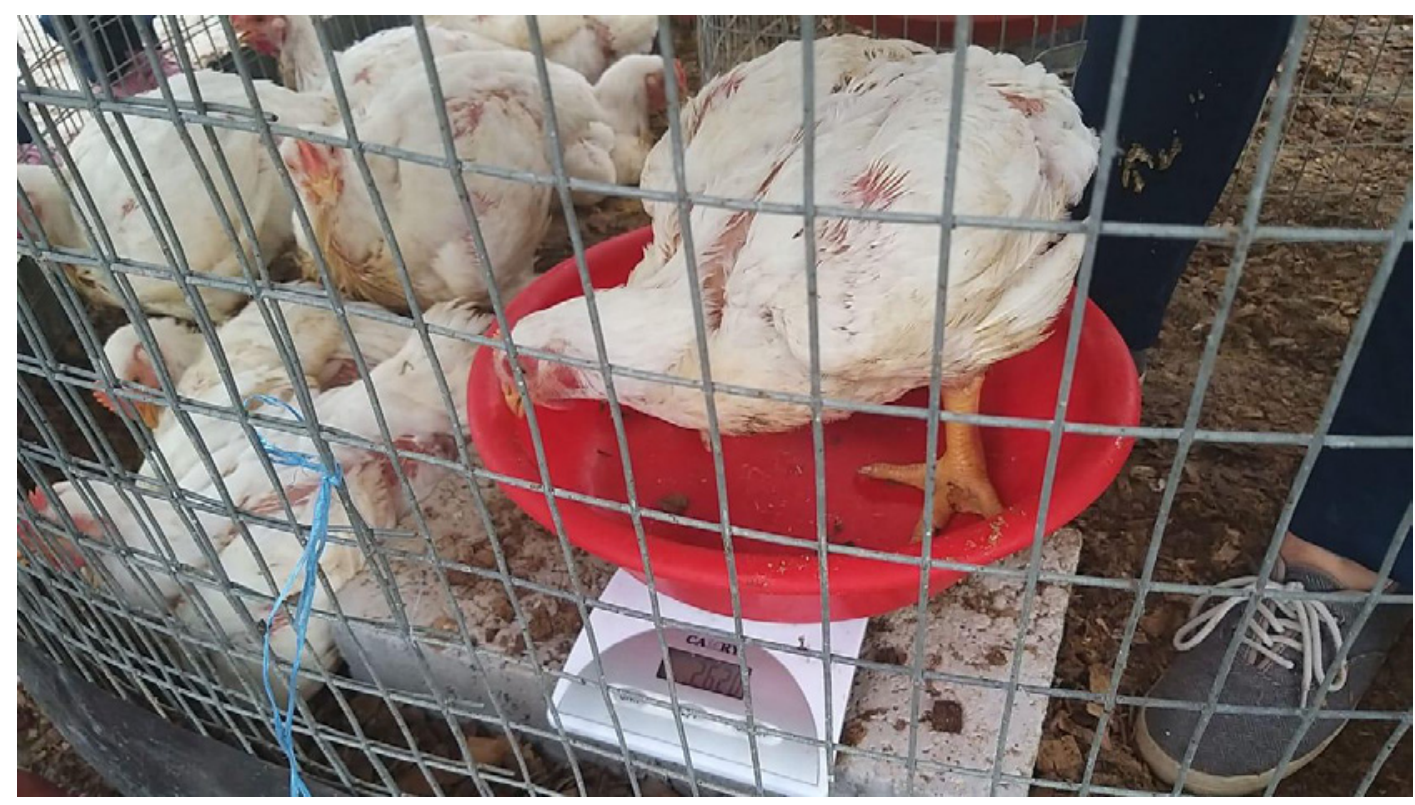

FIGURA 4. Pesaje de pollo COBB 500 
Consumo acumulado de alimento (g): El consumo del alimento se determinó semana a semana, generando 24 datos $(2 \mathrm{~S} \times 2 \mathrm{R} \times 6 \mathrm{~T})$, aplicando la ecuación [1]:

Consumo de Alimento Acumulado $(g)=$ Alimento ofrecido - Alimento sobrante

Consumo acumulado de agua $(\mathbf{m l})$ : El consumo de agua acumulado se registró semanalmente, para ello se utilizó un recipiente de volumen con medidas mínimas de $50 \mathrm{ml}$, generando 24 datos $(2 \mathrm{~S} \times 2 \mathrm{R} \times 6 \mathrm{~T})$, aplicando la ecuación presente [2]:

Consumo de Agua Acumulada $(\mathrm{ml})=$ Agua ofrecida - Agua sobrante

Conversión alimenticia (C.A): La conversión alimenticia se obtuvo mediante la división del alimento consumido y con el peso adquirido del pollo [3]. Se lo obtuvo semanalmente, generando 24 datos $(2 \mathrm{~S} \times 2 \mathrm{R} \times 6 \mathrm{~T})$ :

$$
\text { Conversión alimenticia }=\frac{\text { Total de alimento consumido }(g)}{\text { Peso vivo final }(g)-\text { Peso vivo inicial }(g)}
$$

Mortalidad (\%): Se calculó al contabilizar las aves iniciales, menos las aves que finalizaron y el resultado dividido para el número de aves iniciales, y todo llevado a porcentaje, utilizando la siguiente la ecuación [4]:

$$
\text { Mortalidad }(\%)=\frac{\text { Aves iniciales }- \text { Aves finales }}{\text { Aves iniciales }} \times 100
$$

Peso ante mortem: Es el dato obtenido previo a la faena o de sacrificio del pollo (día 40), expresado en gramos, generando 100 datos (2S x 2R x 25P).

Peso de la canal: Para determinar esta variable en gramos, se sometió a las aves a un ayuno de 8 horas, sacrificándolas por dislocación cervical con el correspondiente colgado del animal y corte de la yugular izquierda para provocar un desangre de 2 minutos como mínimo, luego pasa por el proceso de escaldado a una temperatura de $60^{\circ} \mathrm{C}$, durante 30 segundos y posterior desplume y así obtener el dato de la canal (sin vísceras blancas, solo con cabeza, patas, hígado, molleja y corazón). Se generaron 100 datos (2S x 2R x 25p).

Rendimiento de la canal (\%): Este dato se calcula con la siguiente fórmula, en donde el peso de la canal se la contabilizo sin vísceras y peso ante mortem fue tomado con el pollo vivo [5]:

$$
\text { Rendimiento de la canal }(\%)=\frac{\text { Peso de la canal }(g)}{\text { Peso ante mortem }(g)} \times 100
$$




\section{F. Análisis estadístico.}

Los análisis estadísticos se realizaron según Blasco (2010), para ello se aplicó un análisis de Varianza paramétrico (ANOVA simple), previa comprobación de los supuestos de Normalidad y Homogeneidad, sin embargo, las variables que no cumplen con distribución normal fueron sometidas a la prueba de Kruskal-Wallis, para establecer la diferencia entre las medias se usó el procedimiento de comparación múltiple de Bonferroni con un nivel del 95,0\% de confianza, no obstante, para la Data promedio que no presentó réplica, se empleó una estadística descriptiva. Para todo el procedimiento se usó el programa estadístico Statgraphics Centurión XV.I. ${ }^{\circledR}$.

\section{Resultados y Discusión}

En la tabla 2 a partir del día 14 se muestran diferencias estadísticas significativas que se mantiene hasta el final de la investigación, estos resultados son parecidos a los encontrados por De Obaldía \& Perales (2015), quienes en su estudio, donde utilizaron 3024 aves, demostraron que esta diferencia es atribuida al factor sexo del pollo, puesto obteniendo el mayor peso los machos y el menor las hembras a los 32 días, el mayor consumo lo presentaron los machos y el menor las hembras durante todo el ensayo, los machos presentaron mayor ganancia de peso semanal durante todo el ensayo y el menor las hembras. Los datos concuerdan con el estudio realizado por Reyes \& Cedeño (2010), en su experimento que al evaluar 3136 pollos Cobb no sexable ${ }^{\circledR}$ y Arbor Acres Plus $^{\circledR}$ en el día 35 se obtuvo diferencias significativas en el peso de la canal $(P \leq 0.05)$; Los tratamientos con la línea Arbor Acres Plus ${ }^{\circledR}$ machos y hembras tuvieron mayor porcentaje de uniformidad que los mixtos de las líneas Arbor Acres Plus ${ }^{\circledR}$ y Cobb no sexable $^{\circledR}$ debido a que los machos y hembras de la línea Arbor Acres Plus ${ }^{\circledR}$ se manejaron separados. No obstante, Osorio et al. (2016), determina que existe diferencia estadísticamente significativa en el peso de machos y hembras, ( $p$ $<0,05$ ), donde los machos tienen mayor peso que las hembras, sin importar el balanceado que sea suministrado.

TABLA 2. Promedio de peso vivo de los pollos COBB 500

\begin{tabular}{|l|c|c|c|c|c|c|}
\hline SEXO & DÍA 7 & DÍA 14 & DÍA 21 & DÍA 28 & DÍA 35 & DÍA 40 \\
\hline Hembras & $175,25 \pm 3,16^{\mathrm{a}}$ & $462,67 \pm 8,91^{\mathrm{a}}$ & $897,20 \pm 18,00^{\mathrm{a}}$ & $1434,92 \pm 27,83^{\mathrm{a}}$ & $2094,49 \pm 36,90^{\mathrm{a}}$ & $2457,44 \pm 47,14^{\mathrm{a}}$ \\
\hline Machos & $181,35 \pm 3,87^{\mathrm{a}}$ & $489,55 \pm 10,91^{\mathrm{b}}$ & $985,128 \pm 22,33^{\mathrm{b}}$ & $1584,43 \pm 33,80^{\mathrm{b}}$ & $2335,97 \pm 45,22^{\mathrm{b}}$ & $2817,27 \pm 58,50^{\mathrm{b}}$ \\
\hline
\end{tabular}

ab: Es la representación de las diferencias estadísticas $(P<0,05)$ encontradas al comparar con el $S 1$. 
TABLA 3. Promedio de consumos de alimento acumulada en Pollos COBB 500

\begin{tabular}{|l|c|c|c|c|c|c|}
\hline SEXO & DÍA 7 & DÍA 14 & DÍA 21 & DÍA 28 & DÍA 35 & DÍA 40 \\
\hline Hembras & $178,34 \pm 6,46^{\mathrm{a}}$ & $576,50 \pm 73,69^{\mathrm{a}}$ & $1274,67 \pm 231,78^{\mathrm{a}}$ & $2214,70 \pm 233,49^{\mathrm{a}}$ & $3603,99 \pm 294,07^{\mathrm{a}}$ & $4226,76 \pm 250,66^{\mathrm{a}}$ \\
\hline Machos & $187,50 \pm 6,46^{\mathrm{a}}$ & $665,00 \pm 73,69^{\mathrm{a}}$ & $1323,40 \pm 231,78^{\mathrm{a}}$ & $2338,87 \pm 233,49^{\mathrm{a}}$ & $3817,11 \pm 294,07^{\mathrm{a}}$ & $4602,07 \pm 250,66^{\mathrm{a}}$ \\
\hline
\end{tabular}

ab: Es la representación de las diferencias estadísticas $(P<0,05)$ encontradas al comparar con el $\mathrm{S1}$.

TABLA 4. Promedio en $\mathrm{ml}$ de consumos de agua acumulada en pollos COBB 500

\begin{tabular}{|l|c|c|c|c|c|c|}
\hline SEXO & DÍA 7 & DÍA 14 & DÍA 21 & DÍA 28 & DÍA 35 & DÍA 40 \\
\hline Hembras & $567,00 \pm 0,00^{\mathrm{a}}$ & $1550,00 \pm 113,61^{\mathrm{a}}$ & $3083,00 \pm 113,27^{\mathrm{a}}$ & $5825,00 \pm 231,96^{\mathrm{a}}$ & $9661,00 \pm 357,18^{\mathrm{a}}$ & $11626,5 \pm 339,70^{\mathrm{a}}$ \\
\hline Machos & $533,00 \pm 0,00^{\mathrm{a}}$ & $1500,00 \pm 113,61^{\mathrm{a}}$ & $2933,50 \pm 113,27^{\mathrm{a}}$ & $5466,50 \pm 231,96^{\mathrm{a}}$ & $9079,00 \pm 357,18^{\mathrm{a}}$ & $10777,00 \pm 339,70^{\mathrm{b}}$ \\
\hline
\end{tabular}

ab: Es la representación de las diferencias estadísticas $(P<0,05)$ encontradas al comparar con el S1

En la Tabla 3, describe que no existe diferencia estadística significativa en el consumo de alimento acumulado, lo que concuerda con Madilindi et al. (2018), en el cual usaron 1008 pollos Cobb, separados en 462 machos y 562 hembras, donde argumentan que no hubo diferencias significativas en el consumo de alimento acumulado. Pero difiere de la investigación realizada por, Awad et al. (2017) quienes, en el estudio, donde utilizaron 800 pollos de engorde sexados, dividiendo 400 machos y 400 hembras, demostrando que se encontró diferencias en el consumo de alimento acumulado según el sexo, indicando que los pollos de engorde machos tienen los datos más altos.

En el mismo orden, al analizar el consumo de agua acumulado se puede apreciar una diferencia estadística significativa en los últimos días de producción (día 36-40) entre las hembras y los machos, no encontrándose una referencia bibliográfica que permita discutir este resultado.

TABLA 5. Promedio de conversión alimenticia acumulada en pollos COBB 500

\begin{tabular}{|c|c|c|c|c|c|c|}
\hline \multicolumn{1}{|c|}{ SEXO } & DÍA 7 & DÍA 14 & DÍA 21 & DÍA 28 & DÍA 35 & DÍA 40 \\
\hline Hembras & $1,35 \pm 0,07^{\mathrm{a}}$ & $1,37 \pm 0,21^{\mathrm{a}}$ & $1,49 \pm 0,32^{\mathrm{a}}$ & $1,59 \pm 0,15^{\mathrm{a}}$ & $1,76 \pm 0,16^{\mathrm{a}}$ & $1,75 \pm 0,18^{\mathrm{a}}$ \\
\hline Machos & $1,36 \pm 0,07^{\mathrm{a}}$ & $1,50 \pm 0,21^{\mathrm{a}}$ & $1,41 \pm 0,32^{\mathrm{a}}$ & $1,52 \pm 0,15^{\mathrm{a}}$ & $1,67 \pm 0,16^{\mathrm{a}}$ & $1,66 \pm 0,18^{\mathrm{a}}$ \\
\hline
\end{tabular}

ab: Es la representación de las diferencias estadísticas $(P<0,05)$ encontradas al comparar con el $\mathrm{S} 1$

Tras analizar los datos correspondientes a la conversión alimenticia entre machos y hembras, no se encontró una diferencia estadística como tal, lo cual concuerda con el estudio realizado por (Gonzalo \& Gutierrez, 2013), donde utilizaron 3264 aves, de las cuales 1088 fueron machos y 1,088 hembras de la línea Arbor Acres Plus ${ }^{\circledR}$ y 1088 aves no sexables de la línea Cobb ${ }^{\circledR}$, explicando que no hubo diferencia significativa de la C.A. entre los tratamientos desde el día 7 al 28, mientras que la línea Cobb no sexable ${ }^{\circledR}$ tuvo menor C.A. en el día 35 seguida por la línea Arbor Acres Plus ${ }^{\circledR}$. Esto coincide con la Guía de manejo del pollo de engorde Cobb, el cual afirma que 
el principal parámetro de selección genética de esta línea es el bajo índice de conversión alimenticia, presentando buen rendimiento en producción de carne con el menor consumo de alimento, lo cual difiere con Willis (2008), donde se analizaron 540 pollos de engorde sexados, determinando la conversión alimenticia, evidenciando que en el día 42 se presentaron diferencias significativas tanto en machos y hembras, concluyendo que los pollos de engorde permanecieron constantes en el consumo de alimento, provocando una conversión alimenticia más eficiente. La mortalidad registrada en total fue del $4 \%$ (2\% pertenecía al descarte).

TABLA 6. Promedio de peso ante mortem, peso de la canal y $\%$ rendimiento de la canal en pollos COBB 500

\begin{tabular}{|l|c|c|c|}
\hline \multicolumn{1}{|c|}{ SEXO } & P_ANTEM (g) & P_CANAL (g) & REND_CANAL (\%) \\
\hline Hembras & $2457,44 \pm 47,13^{\mathrm{a}}$ & $2073,28 \pm 40,98^{\mathrm{a}}$ & $84,44 \pm 0,51^{\mathrm{a}}$ \\
\hline Machos & $2817,27 \pm 58,5^{\mathrm{b}}$ & $2315,65 \pm 50,86^{\mathrm{b}}$ & $82,12 \pm 0,64^{\mathrm{b}}$ \\
\hline
\end{tabular}

P_ANTEM: Promedio de peso ante mortem. P_CANAL: Promedio del peso de la canal. REND_CANAL (\%): Promedio rendimien-

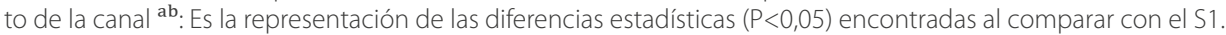

En la tabla 6, se puede observar la diferencia estadística que presenta el pollo macho y hembra, antes y posterior al sacrificio de los mismos, siendo los machos, los que presentaron los datos más relevantes, esta diferencia, es atribuida al factor sexo, puesto que el consumo de alimento aumenta a medida que el ave de engorde crece, mientras que el \% de rendimiento de la canal mostró que la hembra fue mejor en este parámetro. Los datos concuerdan con el estudio realizado por Reyes \& Cedeño (2010), en su experimento de pollos sexado sobre los parámetros productivos y las características de la canal hasta los 35 días de edad que al evaluar 3136 pollos Cobb no sexable ${ }^{\circledR}$ y Arbor Acres Plus ${ }^{\circledR}$ en el día 35 encontraron diferencias significativas en el peso de la canal $(P \leq 0.05)$; la línea Arbor Acres Plus ${ }^{\circledR}$ machos obtuvo un mayor peso que la línea Arbor Acres Plus ${ }^{\circledR}$ mixtos mientras que la línea Arbor Acres Plus ${ }^{\circledR}$ hembras y Cobb no sexable ${ }^{\circledR}$ mixto alcanzaron pesos similares. Con respecto al \% de rendimiento de la canal los resultados fueron distintos a los encontrados por Madilindi et al. (2018), quienes indicaron que en el rendimiento de la canal se encontraron diferencias estadísticas, siendo los machos los que obtuvieron un mayor porcentaje.

\section{Conclusiones}

Al evaluar el efecto de la dieta sin APC, coccidiostatos y aminoácidos sintéticos en los pollos sexados Cobb 500, se encontró que no hay diferencia en la conversión alimenticia y consumo de alimento, pero sí, un efecto marcado en el peso de los animales, siendo los machos los que registran mejores datos a partir de la segunda semana, mientras que las hembras mostraron 
un consumo mayor en el agua de bebida en los últimos días del experimento, dato interesante que debería ser evaluado a profundidad en futuras investigaciones.

El peso de la canal fue mayor en machos en comparación con las hembras, sin embargo, al analizar el porcentaje del rendimiento de la canal las hembras mostraron datos superiores, esto podría explicarse debido a que el macho tiene la tendencia a consumir más alimento y por lo tanto tendrá mayor contenido de este a nivel gastrointestinal.

El alimento administrado a los animales tuvo un efecto en el sexo, los machos presentaron el resultado más notable y en algunos casos diferentes a investigaciones similares pero con otro tipo de dietas que llevan aditivos sintéticos por lo que se debería hacer comparaciones con otros piensos y observar si las diferencias se mantienen.

\section{RECONOCIMIENTO}

A las autoridades de la Facultad de Ciencias Agropecuarias por brindar los espacios para la experimentación y a los alumnos cuarto ciclo de la carrera de Medicina Veterinaria y octavo ciclo de la carrera de Medicina Veterinaria y Zootecnia del año 2020-Dl por el aporte a la investigación de cátedra.

\section{REFERENCIAS}

Agrocalidad (2017). Norma 7.5. Guía General de Carácter Voluntario, referente a la Adopción y Certificación de Buenas Prácticas Avícolas (BPA), Agrocalidad-Ecuador. Obtenido de Articulo 30 Del suministro de alimentos: De los fármacos y promotores de crecimiento en el alimento: https://www.agrocalidad.gob.ec/ wp-content/uploads/2020/05/pecu4.pdf

Almeida, W. P. (2021). Norma zoosanitaria que establece el reglamento para la conformación, aprobación y el seguimiento de Comités de Ética para la investigación con animales en el Ecuador y bioterios. Obtenido de Agencia de Regulación y Control Fito y Zoosanitario de la República del Ecuador: https://aportecivico.gobiernoelectronico.gob.ec/legislation/processes/34/ draft_versions/56

Applegate, T. J. (2017). Additives in poultry nutrition: probiotics, prebiotics and toxin binders. Practical aspects. Obtenido de http://fundacionfedna.org/sites/ default/files/10_CAPX-Applegate.pdf

Arcila-Lozano, Cynthia Cristina, Loarca-Piña, Guadalupe, Lecona-Uribe, Salvador, \& González de Mejía, Elvira. (2004). El orégano: propiedades, composición y actividad biológica de sus componentes. Archivos Latinoamericanos de Nutrición, 54(1), 100-1 11 . Recuperado en 01 de octubre de 2021, de http://ve.scielo.org/scielo.php?script=sci_arttext\&pid=S0004-06222004000100015\&lng=es\&tlng=es

Awad Elmutaz , Zulkifli Idrus, Soleimani Abdoreza \& Aljuobori Ahmed (2017) Effects of feeding male and female broiler chickens on low-protein diets fortified with 
different dietary glycine levels under the hot and humid tropical climate, Italian Journal of Animal Science, 16:3, 453-461. Obtenido de: https://www.tandfonline.com/action/showCitFormats?doi=10.1080\%2F1828051X.2017.1291288 DOI: $10.1080 / 1828051 X .2017 .1291288$

Blasco, A. (2010). Análisis de datos experimentales para proyectos fin de carrera. Departamento de Ciencia Animal. Escuela Técnica Superior de Ingeniería Agronómica y del Medio Natural. Universidad Politécnica de Valencia. ref. 2010.288. Editorial de la UPV.

Caivinagua, J. L. (2016). Efecto de la infusión de oreganon en los parámetros productivos y, como reemplazo del coccidiostato del alimento en pollos broilers. Obtenido de http://repositorio.utmachala.edu.ec/bitstream/48000/7697/1/ DE00050_TRABAJODETITULACION.pdf

Chiriboga Chuchuca, C., Sánchez Quinche, Á., Vargas González, O., Hurtado Flores, L., \& Quevedo Guerrero, J. (2015). Uso de Infusión de oreganón Plectranthus amboinicus (Lour.) Spreng y del vinagre en la crianza de pollos "Acriollados" (Gallus gallus domesticus) mejorados. Obtenido de https://www.redalyc. org/pdf/1699/169944104014.pdf DOI: http://dx.doi.org/10.15446/acag. v65n3.46222

Cobb - Vantress. (2018). Suplemento informativo sobre rendimiento y nutrición de pollos de engorde Cobb 500. Obtenido de Objetivos de Desempeño, Como al Nacimiento, pag 3: https://www.cobb-vantress.com/assets/Cobb-Files/ c8850fbe02/6998d7c0-12d1-11 e9-9c88-c51 e407c53ab.pdf

De Blass, C., García-Rebollar, P., Gorrachategui, M., \& Mateos, G. (2019). Tablas FEDNA de composición y valor nutritivo de alimentos para la fabricación de piensos compuestos. Obtenido de Fundación Española para el Desarrollo de la Nutrición Animal: http://www.fundacionfedna.org/ tablas-fedna-composicion-alimentos-valor-nutritivo

De Obaldía Samudio, J. A., \& Perales Rojas, L. E. (2015). Evaluación de los parámetros productivos entre pollos mixtos, machos y hembras de la línea Arbor Acres plus $^{\circledR}$. (2. Zamorano: Escuela Agrícola Panamericana, Ed.) Obtenido de https:// bdigital.zamorano.edu/bitstream/1 1036/4575/1/CPA-2015-028.pdf

Hegde, NV, Kariyawasam, S. y DebRoy, C. (2016). Comparación de genes resistentes a los antimicrobianos en el microbioma intestinal de pollo cultivado con una dieta orgánica y convencional. Veterinaria y ciencia animal, 1, 9-14. https:// www.sciencedirect.com/science/article/pii/S2451943X16300047 DOI:https:// doi.org/10.1016/j.vas.2016.07.001

Kumar, S., Chen, C., Indugu, N., Werlang, G. O., Singh, M., Kim, W. K., \& Thippareddi, H. (2018). Effect of antibiotic withdrawal in feed on chicken gut microbial dynamics, immunity, growth performance and prevalence of foodborne pathogens. PloS one, 13(2), e0192450. https://journals.plos.org/plosone/article?id=10.1371/journal.pone.0192450 CON DOI https://doi.org/10.1371/journal.pone.0192450

Gerardo, C., González García, E., Flores, C., Carro, M., \& Alban, E. (4 de junio de 2020). Alternativas a los antibióticos de uso alimentario en rumiantes: probióticos, enzimas y ácidos orgánicos. Recuperado el 2003, de Avances en nutrición y alimentación animal: https://hal.archives-ouvertes.fr/hal-01600239/document 
González, C., \& Gutiérrez, E. (2013). Evaluación de la productividad de pollos de engorde de las líneas Arbor Acres Plus ${ }^{\circledR}$ y Cobb no sexable ${ }^{\circledR}$. Obtenido de https://bdigital.zamorano.edu/bitstream/1 1036/1640/1/CPA-2013-041.pdf

Gutiérrez Ramírez, L., Montoya, O., \& Vélez Zea, J. (2013). Probióticos: una alternativa de producción limpia y de remplazo a los antibióticos promotores de crecimiento en la alimentación animal. Obtenido de http://repository.lasallista. edu.co:8080/ojs/index.php/pl/article/view/444

M.A. Madilindi M.A. Letwaba, Tshilate, Banga, Rambau, Bhebhe, Benyi (2018). Effects of sex and stocking density on the performance of broiler chickens in a sub-tropical environment. African Journal Online. Vol. 48 No. 3. Obtenido de: https://www.ajol.info/index.php/sajas/article/view/172383.DOI:10.4314/ sajas.v48i3.6

Fundación Española para el Desarrollo de la Nutrición Animal Necesidades nutricionales para avicultura (2018) NORMAS FEDNA (2 edición) Madrid, 22 y 23 de noviembre Editorial: ISBN: 978-84-09-06529-5. http://www.fundacionfedna. org/sites/default/files/NORMAS_FEDNA_AVES_2018v.pdf

Osorio JH, Quenán YE y Ramírez GF. (2016). Concentraciones de glucemia e insulinemia en pollos broilers machos y hembras de cuatro semanas de edad y su relación con el peso. Rev Med Vet. ;(32): 21-28. Obtenido de: https://ciencia. lasalle.edu.co/cgi/viewcontent.cgi?article=1241\&context=mv DOI: https://doi. org/10.19052/mv.3852

Osorio P., C., Icochea D., E., Reyna S., P., Guzmán G., J., Cazorla M., F., \& Carcelén C., F. (2010). Comparación del rendimiento productivo de pollos de carne suplementados con un probiótico versus un antibiótico. Revista De Investigaciones Veterinarias Del Perú, 21(2), 219-222. https://doi.org/10.15381/rivep.v21i2.140

Pérez, I., Solórzano Saldarriaga, J., Sánchez Quinche, A., Quevedo Guerrero, J., \& Paladines Romero, J. (2019). Effect of Mentha spicata L. Infusion on the productive performance and organoleptic characteristics of Cobb 500 broilers. Obtenido de https://revistas.unal.edu.co/index.php/acta_agronomica/article/ view/72174 DOI: https://doi.org/10.15446/acag.v68n4.72174

Pindo Nagua, F., \& Sánchez Quinche, A. (2016). Uso de zeolita en el alimento como mejorador de los índices de producción de pollos en el cantón Chilla. Obtenido de http://186.3.32.121/bitstream/48000/7698/1/DE00051_TRABAJODETITULACION.pdf

Reyes Carpio, W. X., \& Cedeño Montenegro, J. E. (diciembre de 2010). Evaluación comparativa entre las líneas de pollos Cobb no sexable ${ }^{\circledR}$ y Arbor Acres Plus ${ }^{\circledR}$ sobre los parámetros productivos y las características de la canal hasta los 35 días de edad. Obtenido de https://bdigital.zamorano.edu/bitstream/1 1036/628/1/ T3020.pdf

Rueda, G. A. (2017). Efecto de la infusión de Lippia alba en los parámetros productivos y control bacteriano en pollos de engorde. Obtenido de http:// repositorio.utmachala.edu.ec/bitstream/48000/10537/1/DE00005_ TRABAJODETITULACION.pdf

Sánchez, A., Solórzano Saldarriaga, J., Caivinagua, J., Quevedo Guerrero, J., \& Vargas Gonzales, O. (2016). Efecto de las infusiones de Mentha spicata y Plectranthus amboinicus en la grasa abdominal de pollos. Obtenido de http://investigacion. utmachala.edu.ec/proceedings/index.php/utmach/article/view/201 
Santoma, G., \& Mateos, G. (2018). Necesidades Nutricionales en Avicultura. Obtenido de Normas FEDNA 2da Edicion: http://www.fundacionfedna.org/sites/default/ files/NORMAS_FEDNA_AVES_2018v.pdf

Shang Y, Kumar S, Oakley B y Kim WK (2018) Chicken Gut Microbiota: Importance and Detection Technology. Frente. Veterinario. Sci. 5: 254. doi: 10.3389 / fvets.2018.00254 https://www.frontiersin.org/articles/10.3389/ fvets.2018.00254/full. DOI: https://doi.org/10.3389/fvets.2018.00254

Valenzuela, F Carvallob, MS Moralesa, P Reyesa (2015). Efecto del uso de ensilado seco de salmón en dietas de pollos broiler sobre parámetros productivos y calidad sensorial de la carne. Obtenido de: https://scielo.conicyt.cl/scielo.php?pi$\mathrm{d}=$ S0301-732X2015000100010\&script=sci_arttext\&tlng=e DOI: http://dx.doi. org/10.4067/S0301-732X2015000100010

Willis, W.L (2008). Investigating the Effects of Dietary Probiotic Feeding Regimens on Broiler Chicken Production and Campylobacter jejuni Presence. Obtenido de: https://www.sciencedirect.com/science/article/pii/S0032579119388327. DOI: https://doi.org/10.3382/ps.2006-00458 